\title{
Galaxy Dynamics and the PNe Population
}

\author{
Nigel G. Douglas \\ Kapteyn Astronomical Institute, Postbus 800, 9700AV Groningen, The Netherlands
}

Summary. This review attempts to place the observations of extragalactic planetary nebulae in the context of galactic dynamics. From this point of view only the radial velocities of the $\mathrm{PNe}$ is important. We have built a specialised instrument to detect $\mathrm{PNe}$ in distant galaxies and measure their radial velocities in one step. This is explained in some detail, along with classical techniques for obtaining kinematic information. The review includes a vision of possible future developments in the field.

\section{Spiral Galaxies}

Many of the dynamical properties of galaxies are most easily seen in the case of spirals. Their defining property, which is a bright stellar population in coherent rotation, makes it relatively easy to determine the size and (apart from a sign ambiguity) the orientation of the galaxy and its rotation speed as a function of radius (the "rotation curve"). It has been found that a large fraction of the neutral hydrogen rotates along with the stars, allowing sensitive radio telescopes to map the disk to fainter limits (see Fig $⿴$ for a state of the art H I image).

The non-coherent motions of the stars, usually expressed as the standard deviation of the three velocity vectors in cylindrical coordinates, could be determined, at least for bright galaxies, and this gave clues as to the total mass density of the disk. The rotation curve itself provides a measure of the enclosed mass, and these diagnostics lead to the conclusion that in general considerable dark matter must be present. Observations of the neutral gas out to large radius from the centre, where the stellar contribution to the mass density is very small, suggest that a dark matter halo becomes dominant in most cases. Note that much of the progress in understanding spiral galaxies is possible because the geometry and orientation of the galaxy is evident, and to a lesser extent because radio as well as optical observations can be employed. 


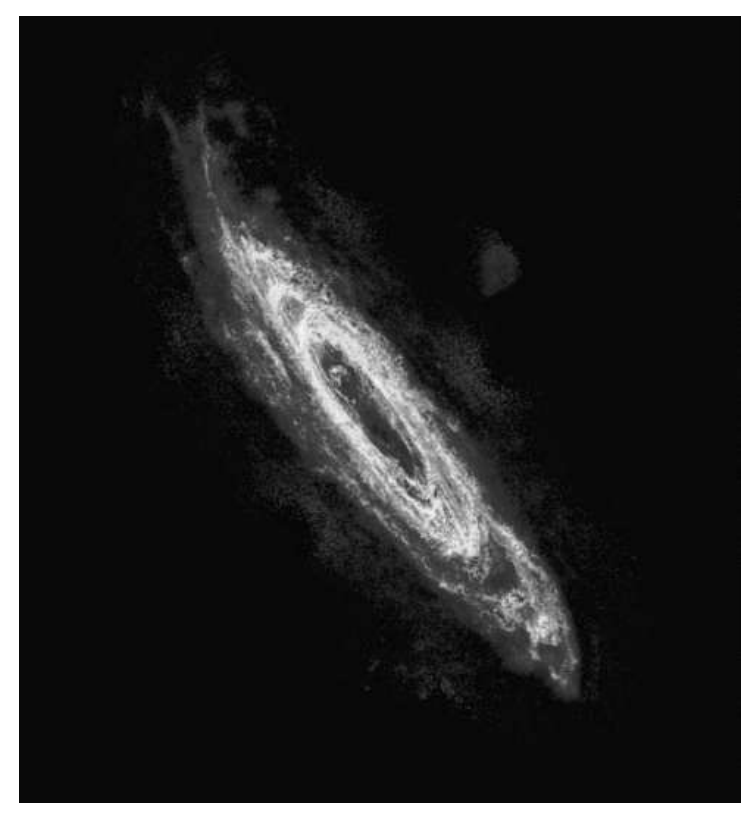

Fig. 1. This radio image of M31 is the most detailed view of an $\mathrm{H} I$ disk ever made, and spans $6^{\circ}$ on the sky. It can be found in 'colour' at 3 .

\section{Elliptical Galaxies}

The situation is quite different in the case of elliptical galaxies (frequently, and unfortunately, referred to as 'early-type' galaxies in the literature). Most of the following remarks apply to the intermediate type S0 galaxies as well. With their characteristic paucity of neutral hydrogen, the kinematics of these galaxies have traditionally been obtained from stellar spectroscopy alone, measuring line-of-sight integrals which are then deconvolved into velocity distributions by template-fitting. The effort involved in doing this is considerable. Moreover, it turns out that the velocity dispersion is usually comparable to, or larger than, any systematic rotation which may be present (see for example Fig 21). This means that, contrary to intuition, rotation alone is not in general responsible for the observed ellipticity of the galaxy. Much of the cause must be sought in incoherent motions, expressed as anisotropy in the velocity distribution of the stars. The anisotropy is difficult to determine from the projected velocity information and the orientation and axis ratio cannot be determined unambiguously from the projected surface brightness. It is difficult even to determine the shape and orientation of the galaxy - an apparently round, spherically symmetric galaxy may be elongated along the line-of-sight and even then this axis can be shorter (oblate galaxy) or longer (prolate) than the tangential axes. Note, in passing, that it can be shown by simple order-of-magnitude arguments, that the optical depth of stars along a random line of sight through a galaxy is small: galaxies 
are transparent. Thus, the integrated spectrum of the starlight contains roughly equal contributions from the "back" and the "front" of the galaxy.

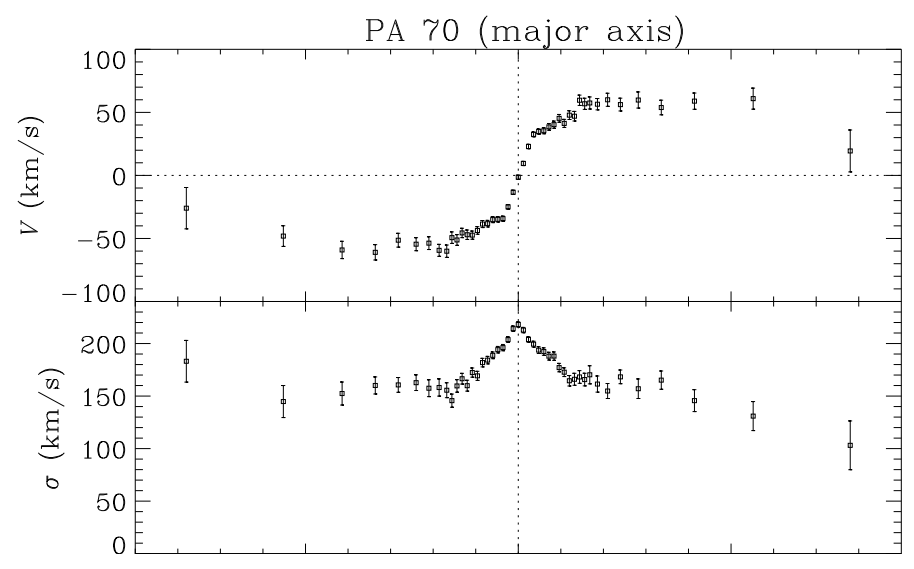

Fig. 2. Major axis rotation in NGC 3379 (from Fig 3a of [19])

Despite these difficulties, integrated light stellar spectroscopy is responsible for virtually all of the basic information that we have on the kinematics of elliptical galaxies. The crucial limitation has turned out to be that, beyond about $2 R_{e}$ (the effective radius $R_{e}$ being the mean radius of a contour containing half of the galaxian light) the surface brightness becomes so low that accurate sky subtraction becomes impossible. The problem is exacerbated for relatively nearby galaxies such as we are talking about here, since the galaxy is so extended that the sky spectrum generally cannot be determined in the same exposure, so that that both spatial and temporal variations degrade the resulting sky subtraction. This means that the outer parts of the galaxies are in effect inaccessible to integrated light spectroscopy. This is unfortunate as it turns out that the circular velocity curves ${ }^{1}$ of nearly all elliptical galaxies are flat, like the rotation curves of spiral galaxies, out to the last measured point, indicating that they too have extended halos. Moreover, some theories of galaxy formation predict that the outer parts of elliptical galaxies possess considerable angular momentum. For this and other reasons we need to be able to explore the kinematics of elliptical galaxies at large radius.

\section{Kinematic Tracers}

One powerful way to circumvent the limitations of integrated light spectroscopy is to look for bright objects in closed orbits in the vicinity of the galaxy - their

\footnotetext{
${ }^{1}$ the $\mathrm{CVC}$ is derived from the sum of the velocity and dispersion in quadrature.
} 
motion gives clues to the gravitational potential of the galaxy and hence to the total enclosed mass. Globular clusters (GC) are one such probe, recent examples being studies in NGC 4472 and Cen A (NGC 5128) 6] 15] respectively. In these galaxies it turns out that the metal-rich and metal-poor GC are kinematically distinct. In the first, the metal-rich GC show net rotation and the metal-poor ones did not, while in the second the converse is true.

Other possible kinematic tracers are satellite galaxies and, in nearby galaxies such as M31 the kinematics of individual stars can be determined (see the article by Ferguson et al. elsewhere in the proceedings).

\section{Planetary Nebulae}

During the workshop, spectacular images of PNe have been presented. Even in the magellanic clouds, the complicated morphology and exhilarating colours of $\mathrm{PNe}$ are easily detected. At greater distances, the PN envelope reduces to an unresolved point and the colours combine to a murky brown. To the extragalactic community, then, the beauty of a PN is only apparent in its spectrum, with its characteristic lines of Hydrogen and 'Nebulium.' The value of PNe as kinematic tracers in external galaxies was demonstrated by Nolthenius and Ford in 1986 [13], combining radial velocity measurements with dynamical modelling of M32. Each PN is seen as a discrete object and it is easy to measure the radial velocity of that object by taking the spectrum. At the specific wavelength of the [O III] emission line (5007 $\AA$ ) a single PN can far outshine the local background of integrated starlight (the emission lines are inherently narrow and usually have a measured linewidth of around $20 \mathrm{~km} / \mathrm{s}$, or $0.3 \AA$, as a result of the expansion velocity of the envelope). $\mathrm{PNe}$ are, in principle, located throughout the body and halo of the galaxy, wherever progenitor stars are to be found. Unlike 'external' probes such as globular clusters, PNe can be expected to share the orbital properties of the underlying stellar population, which means that kinematical information from PNe can be directly combined with stellar kinematics. By 1993 several projects had been completed and the principal theoretical tools were in place, as summarised in a seminal paper by Xiaohui Hui [10. Of special note is the dramatic demonstration, in the case of NGC 3379, of how just a few tens of $\mathrm{PNe}$ can constrain the dynamical models based on integrated light spectroscopy [4.

Comparisons have been made between $\mathrm{PNe}$ and other discrete kinematic tracers. It is interesting that in the cases mentioned in $\$ 3$ the $\mathrm{PNe}$ in Cen $\mathrm{A}$ are found to exhibit significant rotation 14, as do the metal-rich GCs in that galaxy, while data that we have acquired on NGC 4472 shows that the PNe population has little rotation, a different result but again consistent with the metal-rich GCs. The total gravitating mass of Cen A derived from the GCs has also found to be in excellent agreement with the value derived from stellar (PN) kinematics [14.

It is often stated, not least in our own papers [9], that PNe are good tracers of the "old" stellar population. I believe that it is worthwhile keeping in mind that 
there are more assumptions in this statement than is usually realised. The mainsequence lifetime of progenitor stars is not relevant, at least in the case where the galaxy is quiescent with only very low-level continuous star formation. In the steady state the creation rate of $\mathrm{PNe}$ is then independent of main-sequence lifetime, depends only on the initial mass function, and for a typical IMF is as likely to involve a star in the range $2-5 M_{\odot}$ as one in the range $1.5-2 M_{\odot}$. With mean ages of $0.6 \mathrm{GYr}$ and $2 \mathrm{Gyr}$ respectively, these populations may well have different kinematics. What presumably tips the scale in favour of the older stars is the lifetime of the PNe phase, which is a strong function of progenitor mass [21]. On the other hand, PNe formed from high-mass progenitors may be brighter, and hence preferentially detected in surveys. The point is that, in special circumstances, one needs to at least question the veracity of the assumption. The search for possible "young" PNe in Cen A (see Peng's paper elsewhere in this volume) is therefore commendable.

\section{Obtaining Extragalactic PN Velocities}

The traditional technique, pioneered by Hui, Arnaboldi, Ciardullo and others, has been to identify the PNe by means of a two-filter survey. One is a narrowband [O III] filter centred on the systemic velocity of a target galaxy - both PNe and foreground stars show up as bright point-like objects - and the other is a continuum filter in which the stars show up and the PNe do not, or are very faint. This enables PN candidates to be identified, which are then re-observed using fibre or multi-object spectroscopy (MOS) to confirm the identification and to measure the radial velocity from the [O III] line. For sheer volume of effort and persistence over a number of years, the compilation of 780 confirmed $\mathrm{PNe}$ in Cen A must be viewed as one of the exemplary projects using this technique 14. Amongst other results, the existence of $\mathrm{PNe}$ out to $15 R_{e}$ showed that the stellar halo extends that far (see also Peng's contribution elsewhere in these proceedings).

Another noteworthy approach is the use of a Fabry-Perot interferometer, which requires no candidate selection and which identifies the $\mathrm{PNe}$ in the entire field (typically a few arcmin) and returns their velocity (typically with an accuracy of $\sim 30 \mathrm{~km} \mathrm{~s}^{-1}$ ) in one observing run [20].

\section{Slitless Spectroscopy and CDI}

Counter-dispersed Imaging (CDI) is a type of slitless spectroscopy in which antisymmetric images are used to determine velocities. The historical antecedents go back to Fehrenbach (see [9] and references therein) who realised that this procedure not only doubles the effective dispersion, but also diminishes systematic errors. Once again, Cen A plays a role in the story, as it was the first extragalactic object studied using CDI, by modifying an existing instrument at the 
Anglo-Australian Telescope. The idea was to compare PNe velocities and positions found using CDI with those determined earlier by Hui et al. 11] using the survey/fibre technique. The results [7] of the tests were very encouraging: for the $24 \mathrm{PNe}$ found in a certain field the astrometry agreed well with the published data except for three cases, for which there was a discrepancy of more than 1 arcsec, and for precisely these three objects Hui et al. had been unable to measure a velocity. In retrospect, it is clear that the fibres missed the object. CDI, on the other hand, is insensitive to astrometric error and always returns a velocity if the PN is detected. The first 'new' results with CDI on an extragalactic source, using the ISIS spectrograph at the WHT, were published shortly afterwards [8]. The results of slitless spectroscopy at an 8-m telescope are of course dramatic, as exemplified by the detection of 535 PNe in NGC 4697 (see [12] and Méndez' contribution elsewhere in this volume). Amongst other results was a distance determination of around 10.5 Mpc. The relative merits of CDI and other techniques are discussed in [9].

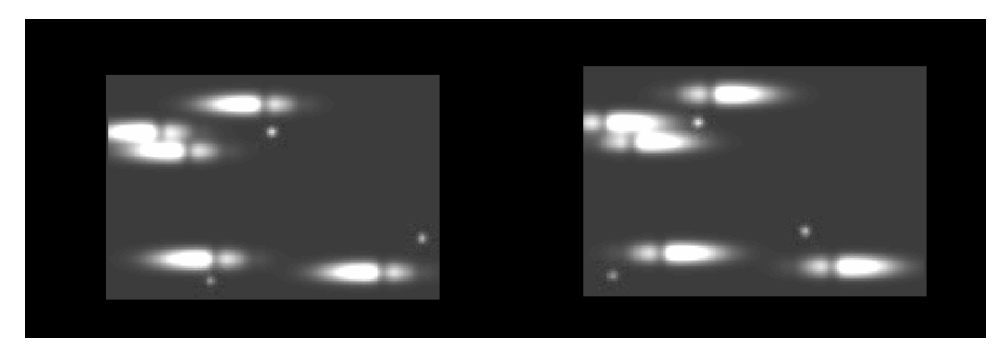

Fig. 3. A simulated pair of CDI images: the extended trails are stellar spectra, with an absorption line, convolved by the [O III] filter profile, while the PNe appear as dots. The illustration is intended to show how, in principle, the relative displacement of the two images of a given PN, in combination with suitably calibrated filter profiles, can return the radial velocity of the PN. In practice calibration is done with a special mask.

\section{The PN.Spectrograph}

The PN.Spectrograph (PN.S), which was developed between 1995 and 2001, is intended to optimally exploit the advantages of CDI described in the previous section. This required (a) that the counter-dispersed images should be obtained simultaneously, not sequentially, (b) that we achieved the highest possible efficiency in the vicinity of the $\lambda 5007$ [O III] line, and (c) that we would make use of a 4-m class telescope, for which relatively large amounts of time are becoming available. Simultaneity of the CDI images is important because any systematic effects (such as seeing) which might affect the centroiding of the PNe images, and hence the velocity measurement, are to first order cancelled out. Moreover one always has a matched set of data, so that one clear night guarantees that 
velocities can be measured, which was not the case with our earlier work [8] and similar programs. Figure 4 shows how we decided to implement simultaneous CDI: a single collimator illuminates an optical pupil in which two gratings are located, edge to edge, each diffracting light into its own camera.

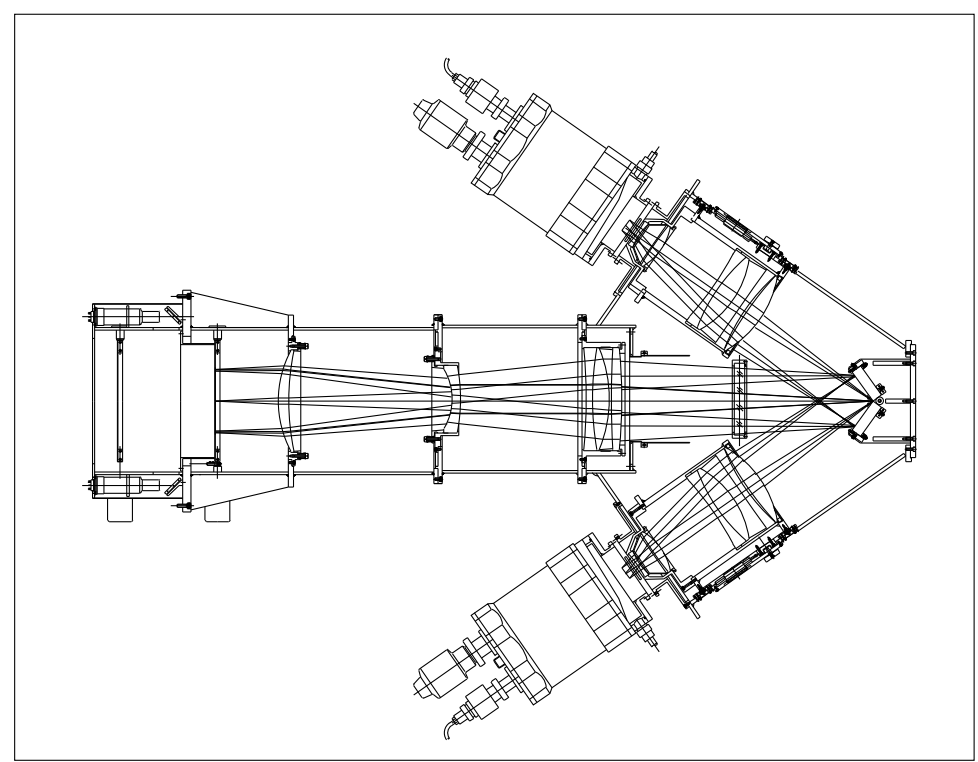

Fig. 4. Schematic of the PN.S - the telescope (not shown) is to the left.

The PN.S was commissioned 2001 after a nail-biting delay caused by the degeneracy of place-names within the Canary Islands - specifically, the instrument was sent to the wrong island. There were however no further problems: the spectrograph worked "straight from the box" with a system efficiency of $33 \%$, including telescope, detector and [O III] filter. For further technical details see 9]. Figure [5 shows the PN.S on the telescope. The first major result with the PN.S was the confirmation of the paucity of dark matter in the outer parts of NGC 3379, as suggested by [4] on the basis of 29 PNe. We have now obtained a total of $400 \mathrm{PNe}$ in three similar galaxies [17. This is part of our core program, namely the study of a sample of apparently round, intermediate-luminosity ellipticals. A side-benefit the PN.S is that effectively all of the observing time is spent in photometric mode, so that the data should yield good luminosity functions and thus distance estimates from the PNLF. On the other hand, the restriction to just the [O III] line makes it difficult to recognise interlopers such as H II regions, which makes the study of later-type galaxies more problematic. To redress this we are in the process of adding a "piggy-back" H- $\alpha$ camera with about the same sensitivity as the main instrument. As has been shown [5], the 
relative flux of these two lines alone provides a useful discriminant against H II regions .

\section{The Accuracy of PNe Radial Velocities}

The limitation on the accuracy with which PNe velocities can be measured is, as usual, set by a combination of the intrinsic linewidth, instrumental accuracy, and the signal-to-noise which is obtained. For example, in the pioneering work of [11, in which Cen A (distance $3 \mathrm{Mpc}$ ) was observed with fibre spectroscopy at the ATT, typical $1 \sigma$ errors were $3-13 \mathrm{~km} \mathrm{~s}^{-1}$, depending on the number of counts obtained $(\sim 50)$. In the case of the PN.S the velocity determination of a given PN depends on the accurate centroiding of each of the two images obtained, as well as, as usual, on the accuracy of the wavelength calibration. We have established the centroiding accuracy which can theoretically be obtained (9], Appendix) and it turns out that for a double detection at the $\mathrm{S} / \mathrm{N}=5$ level the separation of the CDI pairs should be measurable with an accuracy of about 0.6 pixels, corresponding to $20 \mathrm{~km} \mathrm{~s}^{-1}$ with our modest dispersion of 1.0 pixel $\AA^{-1}$. Higher dispersion would help, but CDI does not lend itself to such a choice for reasons discussed elsewhere 9. Initial results, in which we compared measurements of NGC 3379 PNe radial velocities produced by 4 with PN.S commissioning data on the same field, roughly confirm this - apart from a small systematic trend with velocity, which has yet to be investigated, the measurements agreed in absolute heliocentric velocity to an accuracy of $28 \mathrm{~km} \mathrm{~s}^{-1}$, including a contribution of $\sim 7 \mathrm{~km} \mathrm{~s}^{-1}$ from the earlier measurements.

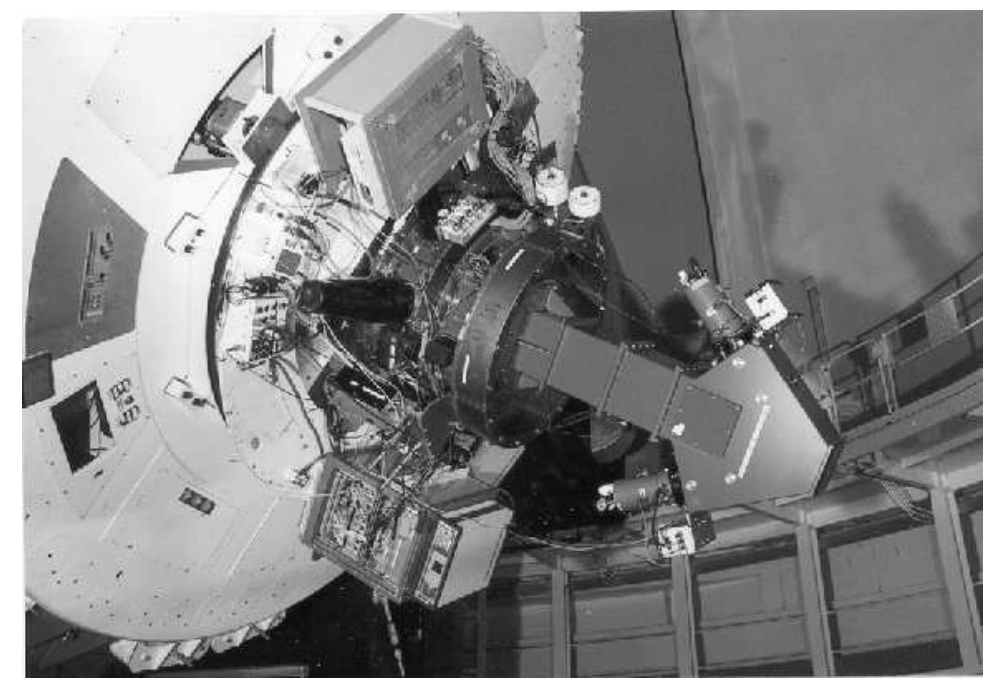

Fig. 5. PN.S at the telescope 


\section{Future Trends}

Encouraged by the speculative discussion initiated earlier in the workshop by Quentin Parker, I present some adventurous predictions with respect to technological advances with the potential to push forward the field of galaxy dynamics.

\subsection{Wavelength-tagged Detectors}

It is surprising how few people are aware of the fact that detectors are potentially capable of registering not only the flux at a given position but also the time of arrival and wavelength of each photon! Some of these properties were indeed realised by the historic Image Photon Counting System (IPCS [2]), but the last parameter, wavelength, remains a challenge. If the wavelength of each photon can be tagged, then we can do away with filters. Any filter bandpass one wishes to specify can be synthesised after the event. The state-of-the-art technology in this area is currently the Superconducting Tunnel Junction (STJ, [16]) which yields a wavelength resolution of about $600 \AA$ in the visible region. This is somewhat better than a V-band filter. Hopefully, with this or perhaps some other technology, we may be able to get down to $60 \AA$ resolution, at which point one could measure the $\mathrm{H} \alpha,[\mathrm{O}$ III], and "off-band" flux from every point in the field, all in one observation, and without having to purchase a single filter (the PN.S filters cost \$US 10,000 each). This technology would rather radically alter our data-collecting strategy.

\subsection{Whole-body IFU}

The integral field unit (IFU) was mentioned during this meeting as an alternative to slit and multi-object spectroscopy in the investigation of extragalactic $\mathrm{PNe}$ (see articles by Exter, and by Roth, and their colleagues, elsewhere in this volume). As such, the IFU must be of potential interest to those of us measuring radial velocities. An IFU consists of more-or-less contiguous cluster of fibres, or else a lens array, covering an area of the field and providing a spectrum of each position. The field size is in principle limited only by the 'real estate', in the form of spectrographs and detectors, that one is prepared to provide. However, this is a non-trivial issue. For galaxy kinematics with PNe one needs to obtain spectra at large galaxian radius and, even though the angular size of each aperture could be made larger to compensate, the number of spectrographs required will still grow quickly. At large galaxian radius, where the background is low, it may be remain cost-efficient to survey for the PNe with two-band images or with slitless spectroscopy. Still, the idea remains exciting since the concept of '3D spectroscopy', the registration of a complete spectrum at each position on the sky, is hard to beat. Fortunately, until such monsters are actually built, there will still be a place for the PN.S. 


\subsection{Tangential Velocities ${ }^{2}$}

However well we measure PNe radial velocities, we are still measuring only one of the three components of the velocity vector. From an analytic point of view this is much worse than measuring the radial velocities for only a third of a sample, say. To illustrate this consider the extreme but not contrived case of an S0 galaxy viewed pole-on. The coherent rotation of the stellar population around the short axis is entirely in the plane of the sky and thus undetectable. In such cases, radial-velocity information alone makes it difficult to unambiguously determine anisotropy however many PNe you detect. Tangential velocities (i.e. proper motions) would lift this ambiguity completely. In the example just given it does not much matter whether the proper motions are determined from the same objects as those whose radial velocities are known, but in general it would be best if all three components could be determined for a set of test particles. Such a test particle then has only one undetermined parameter, its distance along the line-of-sight, whereas if the tangential and radial velocity information is spread over different particles then there are two unknowns.

At first glance, the prospect seems totally beyond the realm of the feasible. Proper motions at extragalactic distances are tiny: at the distance of Cen A (about $3 \mathrm{Mpc}$ ) one could wait approximately 100 years to see 1 milliarcsecond of proper motion. However, the next generation of centimetre-wavelength radio telescopes, of which the Square-kilometre Array (SKA) is the design prototype, together with the existing technology of Very Long Baseline Interferometry (VLBI) may put this within reach.

The performance goals of the SKA translate to a continuum sensitivity over the wavelength range of operation, and for a one hour integration with $512 \mathrm{MHz}$ bandwidth (roughly equal to the internal linewidth of a PN), of $72 \mathrm{nJy}$ beam $^{-1}$. We will see that this is easily sufficient to be able to detect PNe at extragalactic distances, but we ideally also want to be able to detect the same PNe optically to obtain radial velocities. I therefore took the 962 Galactic PNe from the Strasbourg-ESO catalogue [1] for which a reliable [O III] flux measurement was available, and correlated this with the radio catalogue of Siodmiak and Tylenda [18. This catalogue has 264 entries, of which 232 have measured [O III] ( $\lambda 5007$ ) fluxes. Next, I asked which of the PNe would potentially be visible at extragalactic distances. In optical work, a PN magnitude ${ }^{3}$ of $m \sim 28$ is usually the practical limit. Assuming for simplicity that the galactic PNe are at a distance of $10 \mathrm{kpc}$, I shifted them to the distance, indicated by D28 in table 9.3 at which the [O III] flux would be reduced to $m=28$ and calculated the corresponding $5 \mathrm{GHz}$ flux. To be conservative I rejected those PNe for which the flux at D28 was less than $500 \mathrm{nJy}$. This left 47 objects, as listed in the table. No statistical

\footnotetext{
${ }^{2}$ This is work in progress with Jean-Pierre Macquart, until recently working at the Kapteyn Institute and now at the NRAO, Socorro.

${ }^{3} \mathrm{PN}$ magnitudes are defined by eqn(2) in the article by Robin Ciardullo elsewhere in this volume; the apparently arcane zero-point is chosen so that a PN of a given magnitude appears equally bright as a star of that magnitude seen through a standard $\mathrm{V}$-band filter.
} 
conclusions can be drawn from this inhomogeneous procedure, but the point to note is that there exist at least some PNe whose radio and optical fluxes would be detectable out to several Mpc. The question of whether the observation is feasible in practice is still to be investigated - it may be that, at this flux level, confusion rather than sensitivity may be the main problem.

How does the astrometric resolution offered by the SKA compare to that required to observe the transverse motions of planetary nebulae? We would like to measure the transverse velocity of a PN to within $10 \%$, which implies that we measure the angular displacement to the same accuracy. Assume that the typical transverse speed of a planetary nebula is $v \sim 200 \mathrm{~km} / \mathrm{s}$. For a galaxy at a distance $D$ a typical PN then moves an angular displacement:

$$
\Delta \theta=42\left(\frac{T}{1 \mathrm{yr}}\right)\left(\frac{v}{200 \mathrm{~km} / \mathrm{s}}\right)\left(\frac{D}{1 \mathrm{Mpc}}\right)^{-1} \quad \mu \mathrm{as}
$$

over a time interval $T$. If SKA is part of a VLBI network with a $5000 \mathrm{~km}$ baseline we estimate that at $5 \mathrm{GHz}$ and with an attainable $\mathrm{S} / \mathrm{N}$ of 100 we would obtain a positional accuracy of $7 \mu$ as. Note that there is no incentive to improve much on that, since the error in $D$ places a limit of about $10 \%$ on the accuracy to which $v$ can be measured. SKA would reach this accuracy for nearby $(\sim 1 \mathrm{Mpc})$ galaxies in just a couple of years. A more serious concern is the stability of local emission. The appearance or disappearance of local hot spots on the ring could offset the emission centroid significantly. It would be ironic if the beautiful internal structure of $\mathrm{PNe}$, ignored by the galaxy kinematicists, were to return in this way to haunt them.

\section{Acknowledgements}

Thanks to R. Braun of ASTRON for making available Fig 1 to T.S. Statler of Ohio University for helpful comments and for making available Fig 2$]$ and to N. Napolitano for useful discussions. We are grateful for the continuing support of the Isaac Newton Group of telescopes at La Palma.

\section{References}

1. A. Acker, F. Ochsenbein, B. Stenholm, R. Tylenda, J. Marcout, and C. Schohn : Strasbourg - ESO catalogue of galactic planetary nebulae, VizieR Online Data Catalog, (1994)

2. A. Boksenberg, and W. L. W. Sargent : ApJ 198, 31

3. R. Braun, E. Corbelli, R.A.M. Walterbos and D. Thilker : ASTRON Newsletter 17, 3 (October 2002)

4. R. Ciardullo, G. H. Jacoby, and H. B.Dejonghe : ApJ 414, 454 (1993) 
Table 1. Optical and Radio fluxes of some PNe - the columns give name, measured flux at 5007 and $5 \mathrm{Ghz}$, D28 (the distance at which the apparent magnitude at $\lambda 5007$ would be $m=28$ ) and the correspondingly recalculated $5 \mathrm{Ghz}$ flux.

\begin{tabular}{|c|c|c|c|c|c|c|c|c|c|}
\hline Name & $\begin{array}{l}\text { Flux } \\
5007 \AA\end{array}$ & $\begin{array}{c}5 \mathrm{GHz} \\
(\mathrm{mJy})\end{array}$ & $\begin{array}{r}\mathrm{D} 28 \\
(\mathrm{Mpc})\end{array}$ & $\begin{array}{r}5 \mathrm{GHz} \\
(\mu \mathrm{Jy})\end{array}$ & Name & $\begin{array}{l}\text { Flux } \\
5007 \AA\end{array}$ & $\begin{array}{c}5 \mathrm{GHz} \\
(\mathrm{mJy})\end{array}$ & $\begin{array}{r}\mathrm{D} 28 \\
(\mathrm{Mpc})\end{array}$ & $\begin{array}{l}5 \mathrm{GHz} \\
(\mu \mathrm{Jy})\end{array}$ \\
\hline $000.7+03.2$ & $3.90 \mathrm{e}-13$ & 15.6 & 1.4 & 0.80 & $060.5+01.8$ & $5.85 \mathrm{e}-13$ & 26.9 & 1.7 & 0.92 \\
\hline $000.7+04.7$ & $5.87 \mathrm{e}-14$ & 12.8 & 0.5 & 4.36 & $062.4-00.2$ & $6.60 \mathrm{e}-13$ & 17.0 & 1.8 & 0.52 \\
\hline $001.3-01.2$ & $1.80 \mathrm{e}-14$ & 9.6 & 0.3 & 10.68 & $064.7+05.0$ & $8.40 \mathrm{e}-12$ & 245.0 & 6.5 & 0.58 \\
\hline $001.7+05.7$ & $9.12 \mathrm{e}-13$ & 24.4 & 2.1 & 0.53 & 067.9-00.2 & $8.52 \mathrm{e}-14$ & 17.3 & 0.7 & 4.06 \\
\hline $002.8+01.7$ & $9.93 \mathrm{e}-15$ & 13.8 & 0.2 & 27.78 & $088.7+04.6$ & $4.04 \mathrm{e}-14$ & 15.2 & 0.4 & 7.52 \\
\hline $003.6+03.1$ & $1.36 \mathrm{e}-13$ & 23.7 & 0.8 & 3.49 & 093.3-00.9 & $9.38 \mathrm{e}-13$ & 37.3 & 2.2 & 0.80 \\
\hline $005.5+06.1$ & $8.98 \mathrm{e}-14$ & 11.8 & 0.7 & 2.63 & $093.5+01.4$ & $3.93 \mathrm{e}-13$ & 372.0 & 1.4 & 18.94 \\
\hline $006.2-03.7$ & $2.06 \mathrm{e}-13$ & 11.4 & 1.0 & 1.11 & $095.2+00.7$ & $1.07 \mathrm{e}-12$ & 59.9 & 2.3 & 1.12 \\
\hline 008.3-01.1 & $3.66 \mathrm{e}-12$ & 163.6 & 4.3 & 0.89 & $096.3+02.3$ & $5.24 \mathrm{e}-13$ & 16.9 & 1.6 & 0.65 \\
\hline $011.9+04.2$ & $2.51 \mathrm{e}-12$ & 70.5 & 3.5 & 0.56 & $098.2+04.9$ & $1.07 \mathrm{e}-12$ & 28.1 & 2.3 & 0.52 \\
\hline $015.9+03.3$ & $6.29 \mathrm{e}-14$ & 58.6 & 0.6 & 18.62 & $232.8-04.7$ & $1.45 \mathrm{e}-13$ & 26.1 & 0.9 & 3.61 \\
\hline 016.4-01.9 & $1.77 \mathrm{e}-12$ & 78.4 & 3.0 & 0.89 & 235.3-03.9 & $4.02 \mathrm{e}-13$ & 22.2 & 1.4 & 1.10 \\
\hline 020.9-01.1 & $5.33 \mathrm{e}-13$ & 249.0 & 1.6 & 9.34 & $253.9+05.7$ & $1.99 \mathrm{e}-13$ & 6.0 & 1.0 & 0.60 \\
\hline $024.1+03.8$ & $7.75 \mathrm{e}-13$ & 49.1 & 2.0 & 1.27 & $355.9+02.7$ & $4.44 \mathrm{e}-14$ & 21.6 & 0.5 & 9.74 \\
\hline $024.8-02.7$ & $4.93 \mathrm{e}-14$ & 13.6 & 0.5 & 5.51 & 356.5-03.9 & $4.47 \mathrm{e}-14$ & 11.1 & 0.5 & 4.96 \\
\hline $027.6+04.2$ & $5.00 \mathrm{e}-13$ & 20.9 & 1.6 & 0.84 & $358.3+03.0$ & $1.25 \mathrm{e}-14$ & 6.9 & 0.2 & 11.06 \\
\hline $034.0+02.2$ & $1.21 \mathrm{e}-13$ & 31.0 & 0.8 & 5.10 & $358.5+05.4$ & $2.31 \mathrm{e}-12$ & 280.0 & 3.4 & 2.43 \\
\hline $043.1+03.8$ & $1.20 \mathrm{e}-13$ & 21.3 & 0.8 & 3.54 & $358.6+01.8$ & $1.35 \mathrm{e}-13$ & 6.0 & 0.8 & 0.89 \\
\hline 045.9-01.9 & $1.37 \mathrm{e}-14$ & 9.1 & 0.3 & 13.29 & $358.9+03.2$ & $4.67 \mathrm{e}-15$ & 27.3 & 0.2 & 117.01 \\
\hline $048.1+01.1$ & $3.29 \mathrm{e}-13$ & 14.2 & 1.3 & 0.86 & $358.9+03.4$ & $2.41 \mathrm{e}-13$ & 12.4 & 1.1 & 1.03 \\
\hline $052.9+02.7$ & $3.00 \mathrm{e}-13$ & 17.3 & 1.2 & 1.15 & 359.3-03.1 & $8.20 \mathrm{e}-14$ & 11.4 & 0.6 & 2.78 \\
\hline $055.2+02.8$ & $4.84 \mathrm{e}-13$ & 23.2 & 1.6 & 0.96 & $359.7-01.8$ & $6.83 e-13$ & 21.5 & 1.8 & 0.63 \\
\hline $056.0+02.0$ & $1.14 \mathrm{e}-13$ & 14.6 & 0.8 & 2.57 & $359.8+03.7$ & $3.51 \mathrm{e}-14$ & 15.6 & 0.4 & 8.89 \\
\hline $058.9+01.3$ & $6.68 \mathrm{e}-13$ & 17.2 & 1.8 & 0.51 & & & & & \\
\hline
\end{tabular}

5. R. Ciardullo, J. J. Feldmeier, G. H. Jacoby, R. Kuzio de Naray, M. B. Laychak, and P. R. Durrell : ApJ 577, 31 (2002)

6. P. Côté, D. E. McLaughlin, J. G Cohen, and J. P. Blakeslee : ApJ 591, 850 (2003)

7. N.G. Douglas, K. Taylor: MNRAS 307, 190 (1999)

8. N.G. Douglas, J. Gerssen K. Kuijken and M.R. Merrifield : MNRAS 316, 795 (2000), 328, 1200 (2002)

9. N.G. Douglas, M. Arnaboldi, K.C. Freeman, K. Kuijken, M.R. Merrifield,

A.J. Romanowsky, K. Taylor, M. Capaccioli, T. Axelrod, R. Gilmozzi, J. Hart,

G. Bloxham and D .Jones : PASP 114, 1234 (2002)

10. X. Hui : PASP 105, 1011, (1993)

11. X. Hui, H. C. Ford, K. C. Freeman, and M.A. Dopita, : ApJ 449, 592, (1995)

12. R. H. Méndez, A. Riffeser, R.-P. Kudritzki, M. Matthias, K. C. Freeman, M. Arnaboldi, M. Capaccioli, and O. E. Gerhard : ApJ 563, 135, (2001)

13. R. Nolthenius and H. Ford : ApJ 305, 600 (1986)

14. E. W. Peng, H. C. Ford, and K. C. Freeman : ApJ 602, 685, (2004)

15. E. W. Peng, H. C. Ford, and K. C. Freeman : ApJ 602, 705, (2004) 
16. M. A. C. Perryman, F. Favata, A. Peacock, N. Rando, and B. G. Taylor : A\& A 346, L30 (1999)

17. A.J. Romanowsky, N.G. Douglas, M. Arnaboldi, K. Kuijken, M.R. Merrifield, N.R. Napolitano, M. Capaccioli, and K.C. Freeman : Science 301, 1698 (2003)

18. N. Siodmiak, and R. Tylenda : Radio emission from planetary nebulae, VizieR Online Data Catalog (2001)

19. T. S. Statler and T. Smecker-Hane : AJ 117, 839 (1999)

20. B. Tremblay, D. Merritt, and T. B. Williams : ApJL 443, L5 (1995)

21. E. Vassiliadis and P.R. Wood : ApJS 92, 125 (1994) and priv. comm, P. Wood (1999) 